\title{
Spotted around the web: Dementia risk, sleep anxiety, novel variant of NBEA
}

\author{
BY JILL ADAMS, LAURA DATTARO
}

20 AUGUST 2021

WEEK OF AUGUST $16^{\text {TH }}$

\section{Research roundup}

- Autistic adults are more likely to have dementia by age 65 than non-autistic adults, according to an analysis of Medicaid records. Autism Research

- Mice missing the TSC1 gene, which is involved in tuberous sclerosis complex, in striatal neurons are unusually quick to learn a motor task. Cell Reports

- Parents have described their children's autism traits as increasingly disabling since the 1990s. The Journal of Child Psychology and Psychiatry

- Many measures designed to assess everyday skills in young children with neurodevelopmental conditions are so sharply focused on diagnostic traits that they may miss other problems. Autism

- Microglia play a role in neurodevelopmental conditions associated with premature birth, a new review suggests. Journal of Neuropathology \& Experimental Neurology

- Autistic children have more sleep problems, including sleep anxiety, than non-autistic children do, according to a large study in China. Spectrum has covered sleep problems in autism previously. BMC Psychiatry

- A novel variant of the NBEA gene, associated with neurodevelopmental delay and epilepsy, seems to be functionally equivalent to the gene's deletion. Molecular Genetics and Metabolism

\section{Science and society}

- Tech company Google has launched a program to increase hiring and support for autistic employees. Disability Scoop

- The Autism Science Foundation has announced a new two-year fellowship for recent college graduates to gain research experience. Autism Science Foundation

- More women are launching research careers today than 20 years ago, yet fewer stay in 


\section{Spectrum | Autism Research News}

https://www.spectrumnews.org

science compared with men, and they publish fewer papers. Nature

- Proponents of 'dolphin therapy' for autism admit there is no science behind the approach, but they still profit from selling their services to vulnerable children. National Geographic

- A group of U.S.-based scientists has called for reducing travel restrictions for visa-carrying researchers from other countries. Spectrum covered the chaos caused by such restrictions earlier in the pandemic. Nature

- Changes in health insurance for U.S. military members and their families may limit access to applied behavior analysis in nonclinical settings. Spectrum has reported on how limited access is to the common therapy in some states. The Fayetteville Observer 